\title{
Integration of Product Design Process and Task Management for Product Data Management Systems
}

\author{
Rui Lu, Wu-liang Peng and Cheng-en Wang \\ Key Laboratory for Process Industry Automation, Ministry of Education, Northeastern \\ University, Shenyang 110004, Liaoning, P.R. China \\ luruihappy@163.com peng-wuliang@163.com wangc@mail.neu.edu.cn
}

\begin{abstract}
Product design is an evolving process which is characterized with creativity and uncertainty. The task management in this process is different from traditional ones because it involves more specific factors such as user specifications, product design specifications, reengineering, design process, and design data. In this article, we aim to analyze product design process with the viewpoint of project management and task management, and propose an integrated approach to interconnect those constraints, functional and nonfunctional factors coming from both product design and task management processes. In the presented approach, utilizing workflow technique, the design process and task management are directly and closely interconnected at process and data level by using the shared model and the shared database. Therefore, we can easily obtain the work breakdown structure (WBS) for project management. Implemented as part of PDM (Product data management) systems, the proposed method has been tested that it can facilitate the product design.
\end{abstract}

Keywords: Project Management, Workflow, Product data management systems, Iteration, Product design process, Task management

\section{INTRODUCTION}

As the early stage of the product development, product design is becoming vital to the prosperity of enterprise and is critical in product development lifecycle. In order to develop high quality and high performance products with short time-to-market, management of product design information is paramount. As an evolving process of problem solving, design process is characterized with creativity and uncertainty. This tends to iteration in design (see figure 1), i.e. having to go back to earlier stages to correct mistakes when the design choices were flawed or impossible to match the specifications. As the product developments become more and more complicated, the hundreds of thousands of design information generated from product design, and more constraints (management, prototyping and organization) involved in design process are more than any single individual can handle. In a typical product development lifecycle, CAD/CAM tools, PDM systems and Workflow Management Systems are used to follow a design-prototype-implement cycle. On the other hand, Product design implies the definition of a project, the identification of the

Please use the following format when citing this chapter:

I.u, Rui. Peng. W., Wang, C. 2007, in IFIP International Federation for Information Processing. Volume 254, Rescarch and Practical Issues of Enterprise Information Systems II Volume I, eds. I. Xu, Tjoa A., Chaudhry S. (Boston: Springer), pp. 207-218. 
technologies to be used, the establishment of a schedule, an evaluation of the load, communicating with the clients [1]. A Product design process composes several successive stages and is a complex task involving more and more disciplines coming from functional and non-functional aspects. Task management is an important feature of design frameworks [2]. A proper task management approach can facilitate the design process and eventually improve the efficiency of product development.

Product design process and task management are both around enterprise business and producing activity, and they have overlapping and resemblance. Furthermore, product developing project/task always followed by a great business reengineering or great renovations of technology and management, more factors (redesign and reengineering, design data, task resources and their capacities, task execution and monitor, etc.) must be taken into account.

Nightingale [3] provided a framework linking products to innovation processes and described how knowledge, technology and organization are all interrelated. $\mathrm{Li}$ et al. [4] discussed an integrating framework of process and project management and presented the mapping method between them. The CRISTAL system was described in [5] and how to integrate product data and workflow management systems was discussed. There are some other research works reported [6-8]. We have also reviewed some commercial tools. Microsoft Project is often used as general task management. However, it offers limited team support and cannot support information flow. Furthermore it doesn't ties to any single example or domain. Other solutions such as SMARTEAM, TEAMCENTER etc are reviewed too. All of researches and commercial products only focus on partial aspect of design and task process. The design data and task data are only conceptually connected or very loose with little data level commutation and communication. Thus we try to analyze product design with the viewpoint of project management and task management, associate design process with task management, and integrate factors coming from design and task management process to help to design the large-scale complex product.

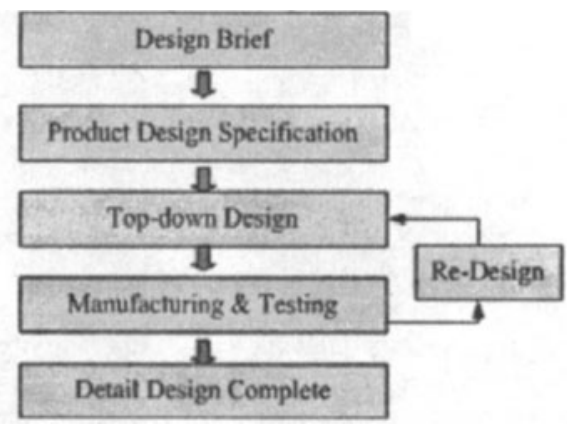

Figure 1. Iterative Product Design Process

In this paper, an approach of integrating the product design process and task management is proposed. The benefits of this solution are that it (a) enables the design process to synchronize with the design task, (b) integrates task information and design data from definition through releasing and execution, (c) facilitates the collection and exchange of design data, (d) facilitates the tracing and monitoring of task management and design process, as well as re-engineering of tasks and processes 
Integration of Product Design Process and Task Management for Product Data

Management Systems 209

by utilizing workflow technique, and (e) associates task with the product specification to ensure the quality of both tasks and processes.

The next section describes the characteristics of task management in product design process and the difference between task management in design process and the typical task management. In the third section, an integrated approach is proposed to interconnect the design and task management processes. This section contains a short introduction to the integrated framework. The proposed approach is described via the general design task management process. The shared database is also described. In the forth section, an integrated task management system is given to illustrate the application of the proposed approach in design projects and design processes. The last section contains the conclusions.

\section{TASK MANAGEMENT IN ITERATIVE PRODUCT DESIGN PROCESS}

The key concept of design process is activities and their sequent relationships. The core of task management is the work breakdown structure and the dependency among tasks. Design Task management system are closely related to product design processes, user specifications, product specifications, design data, design resources, design tasks and task execution and monitoring process[9]. Furthermore, redesign feedback loops can have impact on cost and project schedules. Therefore, reengineering is another important factor should be considered in design task management. Since it is closely related to those factors, the differences between design task management and the traditional one are depicted as following:

a) The outcome of task in product design process is design data, such as product structure profile, graph documents, etc.

b) More constraints or factors coming from design and task management processes should be taken into account. Task management should link functional and non-functional knowledge from two processes.

c) Product data and task data are closely related. Task management should be integrated with design process and eventually integrated with product development lifecycle process.

d) Due to the iteration, which is fundamental characteristic of complex design project, the task duration and task result are stochastic during the task execution.

In manufacturing systems, engineers use PDM to coordinate and control access to documented versions of product designs, and project managers use PM and Task Management tools to control task plan and execution. In the market, few task management factors are currently taken into account by PDM systems although PM and Task Management are considered indispensable function in PDM systems. Our main objective in this paper is to integrate the design process with task management by means of shared model and shared database, and utilize workflow technique to control and monitor both design and task management process. For that, we propose an integrated task management approach which will be described here below. 


\section{INTEGRATED TASK MANAGEMENT APPROACH}

Figure 2 addresses the proposed task management approach and the integration between the product design and task management process by utilizing workflow model. The product design and general design task management processes are illustrated in this figure.

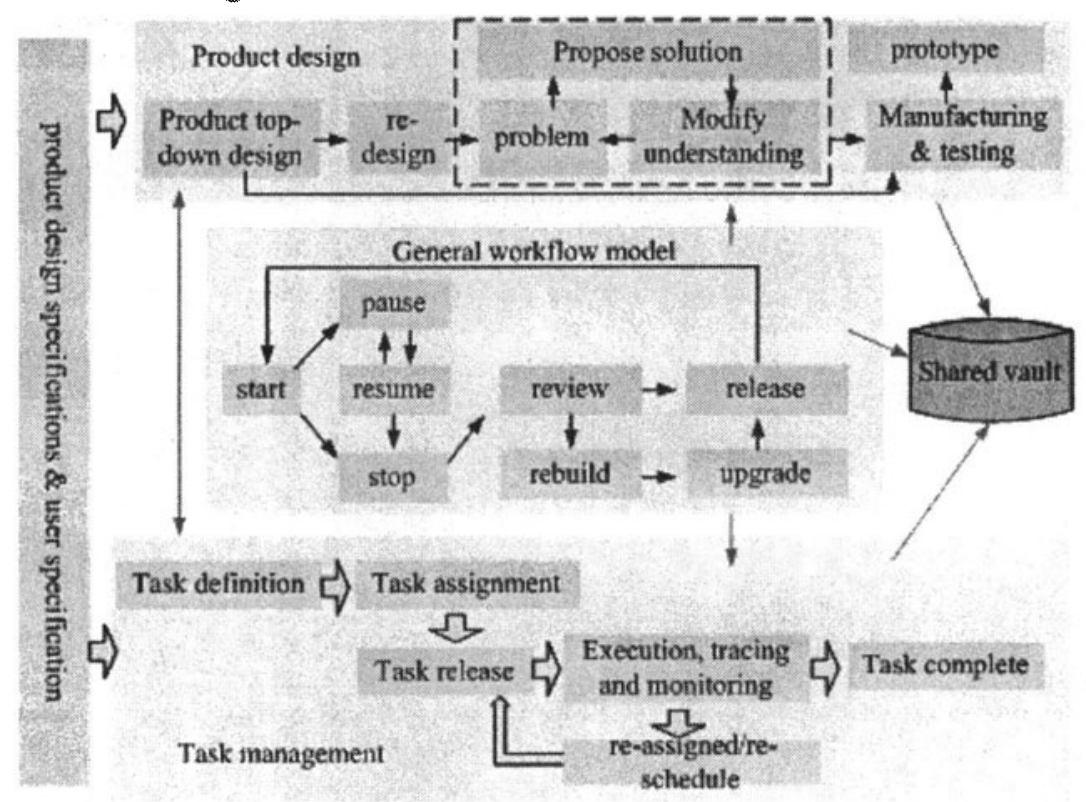

Figure 2. Proposed Approach and its Integration with Design Process

\subsection{The Integrated Framework}

In this framework, product design data and task management information share the same database and the same model. In enterprise, design process is constructed based on experience and experts' knowledge, therefore, is more 'harder'. That means that product design process is not expected to change during the execution of the process. At first, we define a process model with sequence steps in light of the feature of the product design project, product and user specifications, etc. At the same time, several quality activities are appointed to perform at each step. An appointed quality activity consists of design data documents (such as CAD documents), quality documents which is used to control design quality, and reference documents. Those documents are required to download/submit at the start/ end of the certain step. The information model is shown in Figure 3. At every step, workflow models are defined to perform those quality activities automatically during the execution. Then, this rationalized process model is used as the template of task plan. Furthermore, process model is 
used as the basement of process management and is traced and recorded. Once the designer finds that there are some faults during the process execution, he then raises the problems and finds solutions to solve them. Then the process knowledge is fed back and the process reusability can be improved.

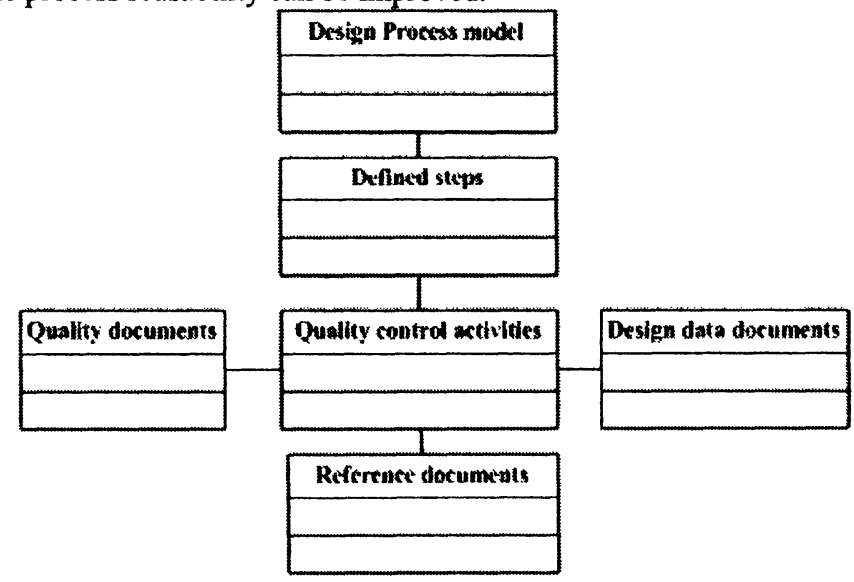

Figure 3. Information Model of Design Process Model

On the other hand, due to the inherent iteration of product development, there are loops that exist in design process. Furthermore, under the execution of tasks, it is common to have a number of unexpected events. As task management does not support conditional branching, recursion or loops, these events are solved by workflow model. For a proper defined workflow instance, it is easy to coordinate the flow of tasks and monitor the collaborative product development process in product development lifecycle. Those defined workflow model can be rebuilt, updated and upgraded and more reasonable and effective service can be supplied.

\subsection{Proposed Task Management Approach}

Hagerman [2] summarized that task management consists of task creation (building a task tree), task resolution/assignment (select tools and data) and task execution (running the tools). In this integrated approach, we propose the general process of task management as steps (task definition, task assignment, task release, task execution, tracing and monitoring, task re-schedule and task re-assignment), as illustrated in Figure 2 .

\subsubsection{Task Definition}

Once the user specifications and product design specifications are finalized and released to design department, design tasks can begin. In the discussed integrated framework, design project managers and task supervisors are authorized to create tasks according to specifications. On the product design side, product structures are hierarchical in nature and product breakdown is often strictly hierarchical in form. 
Attributes can be assigned to each part or sub-part. In industry, product breakdown is often referred to as "Bill of Materials" (BOM). On the more detailed management level in task side, the objective of task creation is to decomposing overall task into hierarchical, smaller, more traceable sub-tasks. The outcome of task definition is Work Breakdown Structure (WBS). The objective of this section is to create the relationship between PBS and WBS in order to help task managers to obtain task tree structure.

Top-down design leads to clear and complete tree-based overall product components or parts view. In reality, PBS represents architecture of components or parts on the side of product design. And the tree structure often implies the design task definition on task management side. This tree-based architecture of tasks is associated with product and its specification and quality control documents. The next step is to map the PBS representation onto design tasks according to functional and nonfunctional parameters. In our framework, this step is called "aggregation/ decomposition and mapping", as shown in figure 4. At this stage of development, at first, design supervisors or managers aggregate and/ or decompose product parts and/ or components according to some parameters (such as function representation, performance, experience and knowledge feedback, reusability of components, qualities, costs and delays, etc). At this stage, the procedure is completely manual and relies on experience obtained in past success and failures. The data coming form the knowledge database provide engineers with the necessary decision supports. The second step consists of defining tasks with three estimated durations-optimistic, most likely, and pessimistic-for their expected duration, creating relations between them, determining delays, costs and tools of them. Following that, those functional representations are mapped onto certain project tasks. At last, we get the work breakdown structure corresponding with product information and quality control documents.

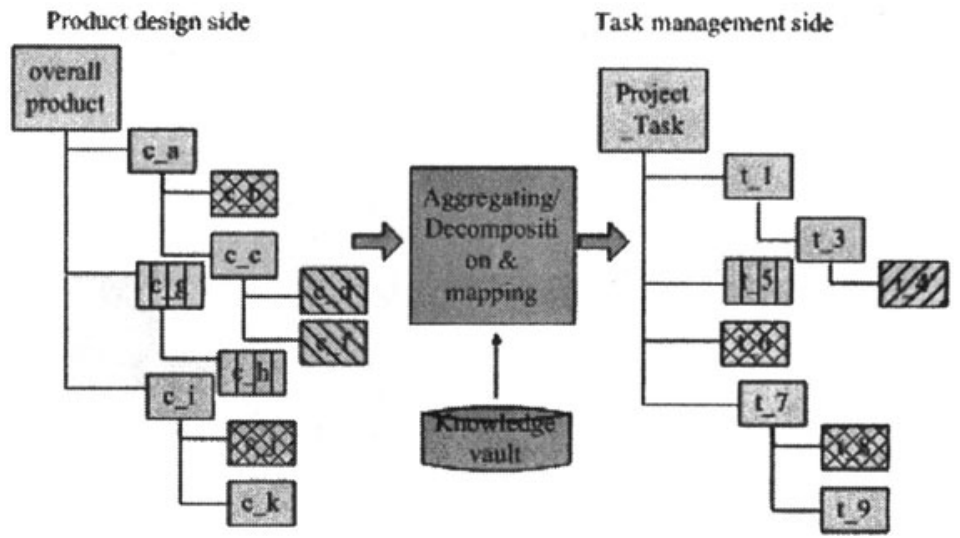

Figure 4. Product Breakdown Structure to Work Breakdown Structure

\subsubsection{Task Assignment}

The team responsible for carrying out the design process is usually being part of the established organization of enterprise, while the designer team responsible for the 
realization of tasks usually disbanded when the design project is completed. In order to integrate the organization with design department, we create project-based organizations which called "design project organizations" in our framework. This organization allows resources to be efficiently allocated in the design project. The defined design tasks are assigned to the designers by design supervisors. One of the benefits of integration of process and task management is to compute the designers' planned workloads from project and resource calendar, and capture their actual daily workloads in real-time from the execution of process. This workload profile will benefit evaluation of task resources' performances. At the same time, designers' workload capacities and availabilities are important parameters that impact on the task schedule. Consequently, they are considered during the task plan and execution and shared during design and task management lifecycle.

\subsubsection{Task Execution and Monitor}

After a task has been assigned to designers, it can be released for execution which means to maintain a schedule, to monitor the plan, to trace the states of task completion and to do re-planning if necessary.

WFMSs as well as PM provide essential functionality for work management (planning and execution support for processes) [10]. In this approach, our aim is to utilize workflow to "mediate" and "glue" the processes, design data and task information as well as different organizations and designers, and eventually to seamlessly integrate the design process and task management. The information model of this approach is illustrated in Figure 5. In this approach, three workflow models (schedule change, quality activities control, and workload calculation workflow model) are defined to monitor and control the task execution.

Quality activities control workflow model: As mentioned previously, the key outcome of design task is design data. In product design process, almost each part or component definition has one or more workflow definition assigned to it to collecting design data, to checking design quality, and to getting approval from supervisor or related organization. We define a workflow model to obtain and control design data created by designers and to monitor information generated from tasks. A FTP service and a relation database are used to contain design data and task information. Designers often submit design documents and data at the end of one stage. The workflow instance is started to perform those functions and return the results. If only the workflow instance return approval, the task can enter the next step through the task flow.

Workload calculation workflow model: As the design process synchronizes with task, a workload calculation workflow model is created to perform the function of tracing and updating the designers' actual workloads and available capacities. The task resource's work load capacity and working performance help to avoid the conflict of task resource assignment.

Schedule change workflow model: Although task plan is made, some of tasks are usually executed in a different order, by unplanned resources with significant and unexpected delays. Due to the quick variations of the environments, as well as the dynamic task schedule change, task execution process is driven by workflow engine. Workflow technology has been reported to be effective in specifying, executing, monitoring, and coordinating the flow of tasks within a distributed environment, 
while reinforcing flexibility [11]. The trigger point of the workflow is the occurrence of an unexpected situation (event) such as overloaded resources, unexpected delays, and etc. As soon as the task is ready to be executed, task schedule workflow instance takes place in the light of the task plan laid out for it. Any changes in the planned schedule can be reflected in the execution of workflow instance without delay. Once the task plan has to be rescheduled, task supervisor should start the workflow instance to run algorithm to do re-schedule, and any changes can be sent to the WFMS. At the same time, task status, such as completion, can be reflected throughout its execution.

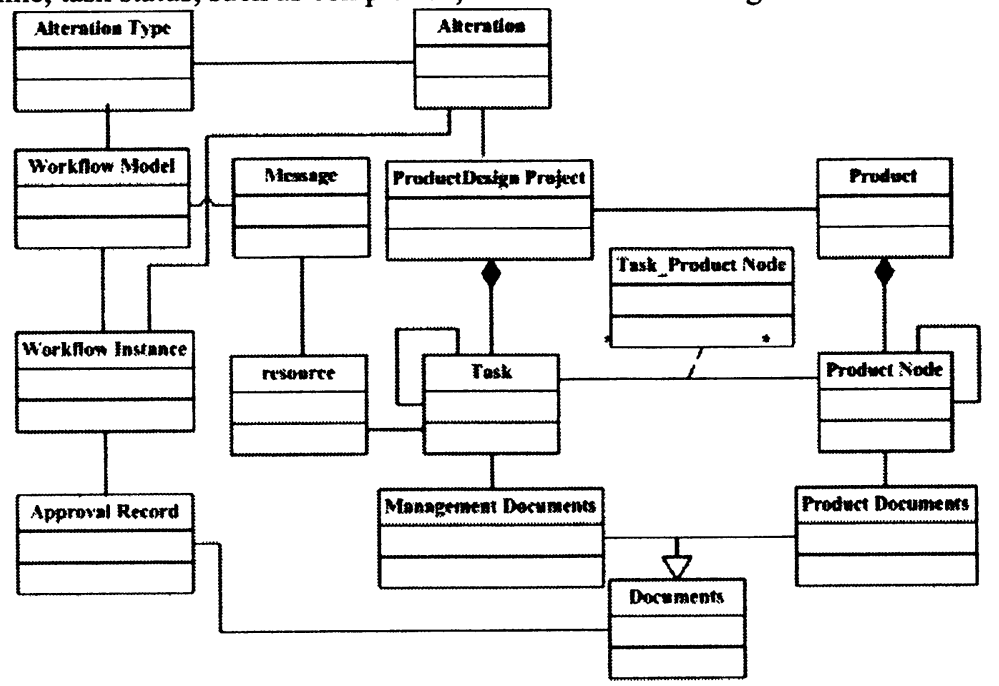

Figure 5. Information Model of Design Task Management

\subsubsection{Task Re-assignment and Re-schedule}

During the execution of the task, when events occur, the schedule sometimes needs to change and may be re-assigned to another designer. For example, if design task $T_{a}$ is not finished after the planned days working, design supervisors decide that the task will continue for several days. Under this circumstance, all related designers (designers of $T_{a}$, as well as the task supervisors of the tasks that have $T_{a}$ as a predecessor) are notified by message sending strategy. The delay of one task will postpone the completion and cause the whole design project delay eventually. Facing this problem, other designers may be assigned to help in $T_{a}$ in order to shorten duration. These events handling procedure is defined as a schedule change workflow model and automatically handled in the proposed approach.

During the execution of design task, two situations of delay (task delay and iteration) could postpone the project. Both of them are considered in our approach. Assuming that task $T_{a}$ is delayed. The effects of the entire project should be recalculated. The procedure is described as two steps: a) tracing for the successors via the chain of dependency relationship until all the affected tasks are identified, and b) revising the time pair (start time and finish time) of all identified tasks. All 
independent tasks are not considered because there is no dependency relationship between them.

As aforementioned, iteration and overlap are important features in product design project. An iterative project network is distinguished from project network without iteration is that the task properties in project is dynamic, meaning that the task sequence is variable, a task duration in each state is stochastic, as well as the task results. When iteration arises, the over-run caused by iteration is usually a fraction of the original duration of task because of each task resource's learning curve [12-14] improvement over the performing stage of the project is considered in our approach. Learning curve measures a characteristic of task when it repeats. The iteration model assumes that the learning curve improves in each repetition until it reaches the minimum fraction of the original duration when a task does the same work repeatedly. Therefore, the time required for later iteration tends to shorter than previous iteration. The duration of tasks that required iteration is re-estimated and computed by the algorithm running in schedule change workflow instance.

Three different re-scheduling methods are used in our approach, namely, revise schedule by averaging, revise schedule manually, and revise schedule based on the weight of tasks [15]. The last method uses numeral $(1 \leq w \leq 10)$ to represent the importance of each task. The amount of time to be shortened for task $T_{a}$ is computed by using equation (1):

$$
t_{i s}=\frac{t_{d}}{\sum_{j=1}^{k}\left(t_{j}^{2} /\left(w_{j} \times \sum_{j=1}^{k} t_{j}\right)\right)} \times\left(t_{i}^{2} / w_{i} \times \sum_{j=1}^{k} t_{j}\right)
$$

Where $t_{i s}$ : the shortened time of the $i$ th task; $t_{d}$ : the overdue time of the entire schedule; $t_{j}$ : the duration of the $j$ th task $(1 \leq j \leq k) ; w_{j}$ : the weight of the $j$ th task $\left(1 \leq w_{j} \leq 10\right)$.

\subsection{The Shared Database}

So far the design and task management are traced by workflow model and become active. And the information flow is also automatic, as all relevant information is stored in the unique database, allowing concurrent multiple authorized users to extract useful information. The shared database stores the static and dynamic data structures of processes and tasks. It contains structural information such as tasks and their relations, planned task duration and resources assigned to tasks. It also stores the dynamic information about the real-time status of tasks and design processes, as well as the historical task records. Considering the design data generated from design task and process combines multiple formats, a FTP server is created to contain the unstructured data such as graph documents.

With the proposed approach, as a product go through its design lifecycle, design process synchronizes with task management process. The design task is linked with 
product and designers' workloads. And the actual workload is updating under the task execution. Product specification, quality and reference documents are directly routed to designers by workflow. Design data and task detail information are collected under the workflow model execution. The integrated approach has been implemented as part of a PDM system; the next section gives scenario of its integrated task management subsystem.

\section{AN INTEGRATED TASK MANAGEMENT SYSTEM}

The integrated system has been deployed as the part of PDM systems. It can facilitate the collection and exchange of design data, reduce the "time-to-market", and improve the efficiency of design process.

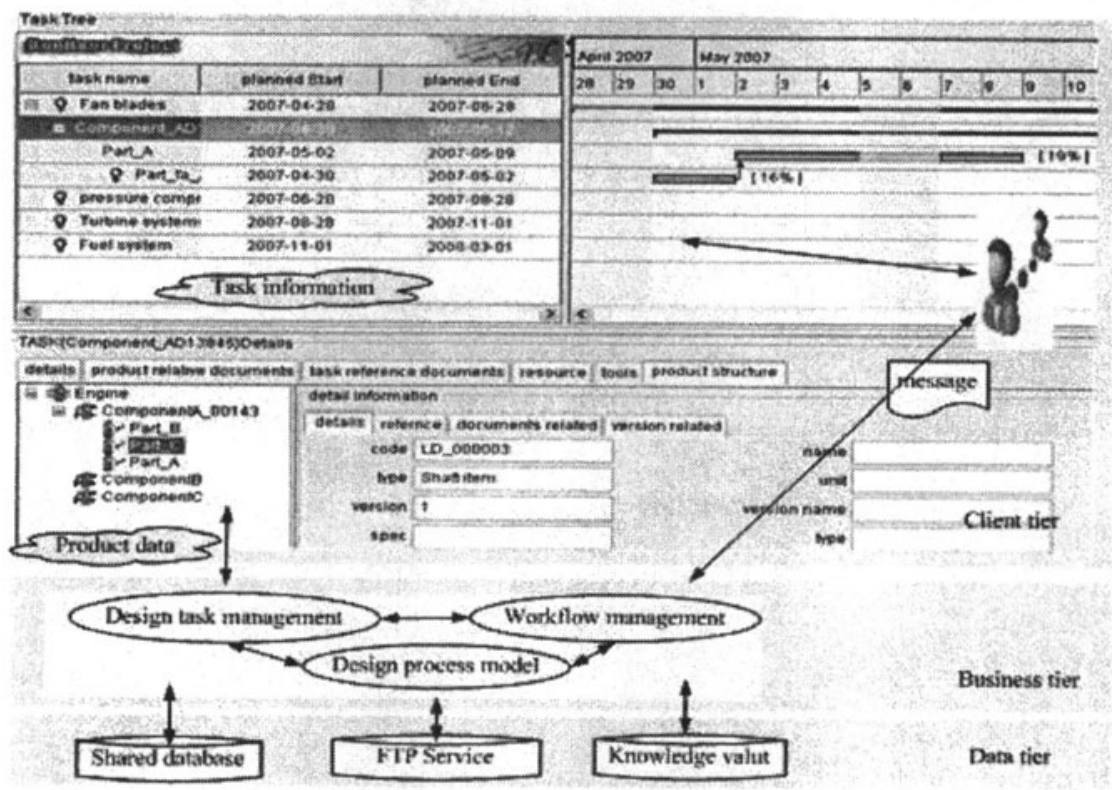

Figure 6. Three-tiered Architecture

The integrated system is built on the J2EE platform. Conceptually, the architecture takes a three-tiered approach (see Figure 6). On the client tier, the workbench provides GUI which comprises design data and task management interface for product definition, task planning and performing. The business logic tier consists of application logic and provides typical services for design process and task management. On the data tier, the data is stored in a SQL Server 2000 database and all design documents generated from design process are managed through FTP service using Microsoft IIS. 


\section{DISCUSSION}

When mapping the PBS representation onto design tasks, human intervention is needed. However, the proposed approach does not support the functionality that can validate the mapping made by engineers. Furthermore, in case of multiple mapping alternatives, there is no method to allow the planner to simulate the entire processes of each alternative. Therefore, the proposed approach can be extended by support the functionality of mapping and simulation.

On the other hand, we assume that task resources and their capacities are sufficient for design project. However, in reality, the resource conflicts are one of the bottlenecks which actually affect the project schedule. So this approach can be further extended by taking variable resource capacity and requirements into account.

\section{CONCLUSIONS}

As the early step of product development, design is critical for enterprise. One strategy for reducing time-to-market development is to take constraints that usually appear during the product design project into account. The main ambition of the research is to consider all functional and non-functional factors coming from both design and task management processes. We proposed an integrated approach to facilitate seamlessly interconnection between product design process and design task management, and thereby providing consistency between design data and task status and information and eventually improving product design efficiency. As related previously, task management during design process is different from the traditional one. The outcome of design task is usually the product structure profile and design documents. Therefore, task is directly related with product node and design data generate from designers. In our approach, workflow technology acts as a "media" and a "glue" to automate the processes design tasks, tracking and monitoring task status, collecting design data emerging from design process and task execution, and triggering defined workflow instance to maintain product quality and feeds experience database with feed-back records which will be benefit for future similar product or product design projects.

\section{REFERENCES}

1. C. Gutierrez, C. Baron, L. Geneste, P. Clermont, D. Esteve, and S. Rochet, How to interconnect Product Design and Project Management including Experience Feedback and Reusability, in Proc. of Information Reuse and Integration, Conf. 2005. IRI-2005 IEEE International Conference (IEEE: 2005), pp.294-299.

2. J.W. Hagerman and S.W. Director, Improved Tool and Data Selection in Task Management, in Proc. of the 33rd ACM/IEEE Design Automation (IEEE: Las Vegas, USA, 1996), pp.181-184.

3. P. Nightingale, The Product-Process-Organization relationship in complex development projects, Research Policy. Volume 29, pp.913-930, (2000). 
4. Q. Li, Y. Chen, and Q. Wang, Integration of Process and Project Management System, in Proc. 2002 IEEE Region 10 Conference on Computers, Communications, Control and Power Engineering, Volume 3 (IEEE: 2002), pp.1582-1586.

5. R. McClatchey, Z. Kovacs, F. Estrella, J-M. Le Goff, G. Chevenier, N. Baker, S. Lieunard, S. Murray, T. Le Flour, and A. Bazan, The Integration of Product Data and Workflow Management System in a Large Scale Engineering Database Application, in Proc. of Database Engineering and Applications Symposium (IEEE: Cardiff, UK, 1998), pp.296-302.

6. V. Bellotti, N. Ducheneaut, M. Howard, and I. Smith, Taking Email to Task: The Design and Evaluation of a Task Management Centered Email Tool, in Proc. of the SIGCHI conference on Human factors in computing systems, Volume 5, Number 1 (IEEE: Florida, USA, 2003), pp.345-352.

7. J. Moore, J. Stader, A. Macintosh, A.C. Mont, and P. Chung, Intelligent Task Management Support for New Product Development in the Chemical Process Industries, in Sixth International Product Development Management Conference (PDM 99) (Cambridge, UK, 1999), pp. 787-796.

8. F. Maurer, B. Dellen, S. Goldmann, H. Holz, B. Kotting, and M. Schaff, Merging Project Planning and Web-Enabled Dynamic Workflow Technologies, IEEE internet computing, IEEE. Volume 4, Issue 3, pp.65-74, (2000).

9. W. He, I.B.H. Lee, E.W. Lee, and W. He, An integrated design Task management approach for product development lifecycle, in Proc. of Industrial Informatics, 2006 IEEE International Conference (IEEE: 2006), pp.554-559.

10. C. Bussler, Workflow Instance Scheduling with Project Management Tools, in Proc. of Database and Expert Systems Applications (1998) (IEEE: Vienna, Austria, 1998), pp.753-758.

11. H.M. Shih and M.M. Tseng, Workflow technology-based monitoring and control for business process and project management, International Journal of Project Management. Volume 14, Number 6, pp.373-378, (1996).

12. T.R. Browning and S.D. Eppinger, Modeling Impacts of Process Architecture on Cost and Schedule Risk in Product Development, IEEE transactions on engineering management. Volume 49, Number 4, pp.428- 442, (2002).

13. E.Z. Huang and S.J. Chen, Estimation of Project Completion Time and Factors Analysis for Concurrent Engineering Project Management: A Simulation Approach, Concurrent engineering: Research and Applications. Volume 14, Number 4, pp.328-341, (2006).

14. S.H. Cho and S.D. Eppringer, A Simulation-Based Process Model for Managing Complex Design Projects, IEEE Transactions on Engineering Management. Volume 52, Number 3, pp.316-328, (2005).

15. C.H. Chen, S. Ling, and W. Chen, Project Scheduling for collaborative product development using DSM, International Journal of Project Management. Volume 21, Number 4, pp.291-299, (2003) 